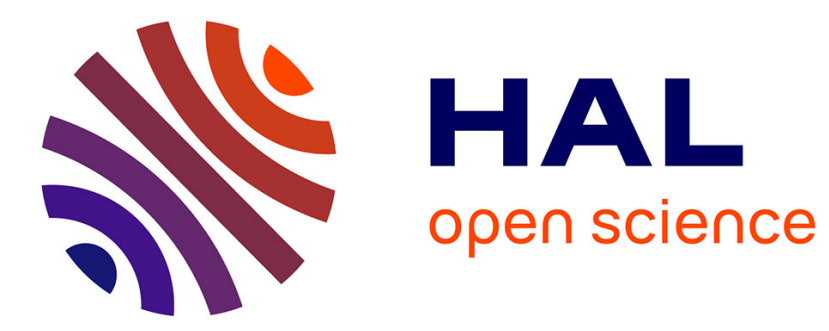

\title{
Sensing a THz electric field with a molecular ion clock Florin Lucian Constantin
}

\section{To cite this version:}

Florin Lucian Constantin. Sensing a $\mathrm{THz}$ electric field with a molecular ion clock. Optical and Quantum Sensing and Precision Metrology, Mar 2021, San Francisco, CA, United States. pp.1170046, 10.1117/12.2591976 . hal-03335652

\section{HAL Id: hal-03335652 \\ https://hal.science/hal-03335652}

Submitted on 23 Sep 2021

HAL is a multi-disciplinary open access archive for the deposit and dissemination of scientific research documents, whether they are published or not. The documents may come from teaching and research institutions in France or abroad, or from public or private research centers.
L'archive ouverte pluridisciplinaire HAL, est destinée au dépôt et à la diffusion de documents scientifiques de niveau recherche, publiés ou non, émanant des établissements d'enseignement et de recherche français ou étrangers, des laboratoires publics ou privés. 


\title{
Sensing a THz electric field with a molecular ion clock
}

\author{
F. L. Constantin*a
}

aababoratoire PhLAM, CNRS UMR 8523, 59655 Villeneuve d'Ascq, France

*f1.constantin@univ-lille1.fr

\begin{abstract}
This contribution proposes a new method for THz-wave detection that exploits the sensitivity to external fields of the transitions of accurate molecular ion clocks. A THz electric field, off-resonantly coupled to the $\mathrm{HD}^{+}$energy levels, may be characterized from the comparison between the measurements of the lighshifts induced on a two-photon rovibrational transition probed on cold trapped molecular ions, and the theoretical predictions of the molecular ion theory. A set of six independent lightshift measurements may be converted into the amplitudes and the phases of the Cartesian components of the $\mathrm{THz}$ electric field in the laboratory frame.
\end{abstract}

Keywords: molecular ion clock, THz sensing, electrometry, two-photon spectroscopy, Zeeman effect, ac-Stark shift, hydrogen molecular ions, cold and trapped molecular ions 


\section{INTRODUCTION}

Precision measurements with simple quantum systems under strict control of the external perturbations play an important role in fundamental physics and enable many technological applications. The Rydberg atoms are particularly sensitive to the electric fields. Detection of microwave photons was performed at the single-photon level with a superconductiong microwave cavity $^{1}$ and the sensitivity was improved using a quantum enhancement technique ${ }^{2}$. Rydberg atomic spectroscopy in vapour cell allowed accurate and stable detection of microwave electric fields with sub-wavelength resolution ${ }^{3}$. This method allowed weak microwave electric field detection at the $\mu \mathrm{V} / \mathrm{cm}$ level with a sensitivity limited by the photon shot noise ${ }^{4}$.

At higher frequencies in the $\mathrm{THz}$ domain, the electromagnetic waves lack of traceable calibration methods. This contribution proposes an approach for traceable detection of a $\mathrm{THz}$ electric field based on measurements of the lighshifts induced by the ac-Stark effect on molecular transitions. The hydrogen molecular ions are the simplest molecular systems. Their energy levels ${ }^{5,6}$ and shifts in external fields ${ }^{7,8,9}$ were calculated accurately by the quantum theory. The spectroscopy of $\mathrm{HD}^{+}$ provided accurate results for the infrared transitions using ion beams ${ }^{10}$ and trapped and sympathetically cooled ions ${ }^{11,12,13}$. Doppler-free spectroscopy techniques with significantly improved accuracy and resolution were demonstrated recently for the fundamental rotational transition of $\mathrm{HD}^{+14}$ and a two-photon infrared transition of $\mathrm{HD}^{+15}$. Combining precision measurements of the frequency shifts of $\mathrm{HD}^{+}$clocks and ab-initio predictions may be exploited for the detection and calibration of the electromagnetic fields. Precisely, a THz-wave off-resonantly coupled to the fundamental rotational transition of $\mathrm{HD}^{+}$at $1.3 \mathrm{THz}$ may be characterized by measurements of the lightshifts induced on a Zeeman component of the two-photon rovibrational transition at 55.9 THz. A set of scalar phase-less lightshift measurements performed in function of the orientation of the quantization axis of the $\mathrm{HD}^{+}$ions may allow full mapping of the THz-wave electric field vector in three dimensions. 


\section{2. $\mathrm{HD}^{+}$MOLECULAR ION THEORY}

\subsection{Energy levels and shifts in external fields for $\mathrm{HD}^{+}$}

The energy levels of $\mathrm{HD}^{+}$are expressed as the sum of the contributions from spin-independent terms corresponding to the rovibrational energy and spin-dependent terms due to spin interactions and to the interactions with the external fields. In the case of the interaction with a static magnetic field B, the energy is expressed as :

$$
E\left(v, L, F, S, J, J_{z}\right)=E_{r v}(v, L)+E_{\text {spin }}\left(v, L, F, S, J, J_{z} ; B\right)
$$

The rovibrational energy $E_{r v}$ is predicted in function of a set of fundamental constants with the Schrödinger equation solution and a series expansion of quantum electrodynamics corrections ${ }^{16}$. The spin structure is due to the coupling of the proton $\vec{I}_{p}$, deuteron $\vec{I}_{d}$, and electron $\vec{S}_{e}$ spins with the rotational angular momentum $\vec{L}$ that yields the total angular momentum $\vec{J}$. The eigenvectors $\mid v L F S J J_{z}>$ are described with the vibrational quantum number denoted $v$, the quantum numbers of the squared angular momenta, and of the projection of the total angular momentum on the quantization axis $J_{z}$. The magnetic energy levels $E_{\text {spin }}$ are calculated by diagonalizing an effective Hamiltonian for each rovibrational state ${ }^{7,17}$, that is expressed as:

$$
\begin{aligned}
& H_{\text {spin }}=H_{h f}+E_{10}(\vec{L} \cdot \vec{B})+E_{11}\left(\vec{I}_{p} \cdot \vec{B}\right)+E_{12}\left(\vec{I}_{d} \cdot \vec{B}\right)+E_{13}\left(\vec{S}_{e} \cdot \vec{B}\right) \\
& H_{h f}=E_{1}\left(\vec{S}_{e} \cdot \vec{L}\right)+E_{2}\left(\vec{I}_{p} \cdot \vec{L}\right)+E_{3}\left(\vec{I}_{d} \cdot \vec{L}\right)+E_{4}\left(\vec{S}_{e} \cdot \vec{I}_{p}\right)+E_{5}\left(\vec{S}_{e} \cdot \vec{I}_{d}\right) \\
& +2 E_{6}\left(L^{2}\left(\vec{S}_{e} \cdot \vec{I}_{p}\right)-3\left(\vec{I}_{p} \cdot \vec{L}\right)\left(\vec{S}_{e} \cdot \vec{L}\right)\right)+2 E_{7}\left(\vec{L}^{2}\left(\vec{S}_{e} \cdot \vec{I}_{d}\right)-3\left(\vec{I}_{d} \cdot \vec{L}\right)\left(\vec{S}_{e} \cdot \vec{L}\right)\right) \\
& +2 E_{8}\left(L^{2}\left(\vec{I}_{p} \cdot \vec{I}_{d}\right)-3\left(\vec{I}_{p} \cdot \vec{L}\right)\left(\vec{I}_{d} \cdot \vec{L}\right)\right)+E_{9}\left(L^{2} \cdot \vec{I}_{d}^{2}+\frac{3}{2}\left(\vec{I}_{d} \cdot \vec{L}\right)-3\left(\vec{I}_{d} \cdot \vec{L}\right)^{2}\right)
\end{aligned}
$$

The values of the coefficients $E_{1} \ldots E_{13}$ are calculated ab-initio in refs. ${ }^{7,17}$.

The interaction with the electromagnetic radiation shifts the $\mathrm{HD}^{+}$energy levels due to the AC-Stark effect. This contribution proposes to exploit the lightshift of an energy level due to a THz-wave offresonantly coupled to another level, which is expressed as :

$$
\delta E_{n}=-\frac{1}{4} \sum_{q=\{-1,0,1\}}(-1)^{q}\left|E_{-q}\right|^{2} \alpha_{n, q}
$$

in function of the amplitudes $E_{q}$ of the standard components of the $\mathrm{THz}$ electric field. The standard dynamic polarizability $\alpha_{n, q}$ is expressed using the second-order perturbation theory in function of the 
matrix elements of the electric dipole operator, the values of the energy levels, and the decay rates of the energy levels ${ }^{18}$. The numerical value and its uncertainty are calculated using a set theoretical parameters $\left\{U_{t h}\right\}$ from refs. ${ }^{7,16,17,19,20}$, the value of the magnetic field measured by Zeeman spectroscopy with $10^{-10} \mathrm{~T}$ level uncertainty (see Section 4), and the frequency of the THz-wave assumed with $10^{-12}$ fractional uncertainty. The fractional uncertainty of the rovibrational energies is assumed at $10^{-12}$. Their radiative lifetimes are at the ms level or better. The hyperfine energies are calculated with $0.5 \mathrm{kHz}$ uncertainty. The fractional uncertainty of the Zeeman shift parameters is assumed at $10^{-4}$ or better. The fractional uncertainty of the electric dipole transition moments is assumed at $10^{-4}$ or better.

\subsection{Two-photon operator formalism for hyperfine levels of $\mathrm{HD}^{+}$}

The two-photon transitions between the ground $|g>=| v_{1} L_{1} F_{1} S_{1} J_{1} J_{z 1}>$ level and the excited $|e>=| v_{2} L_{2} F_{2} S_{2} J_{2} J_{z 2}>$ level are driven with two counterpropagating waves with standard polarizations $p_{1}, p_{2}$, powers $P_{1}, P_{2}$, and section $S$, at the angular frequency $\omega$, slightly detuned to intermediary levels $|r>=| v_{3} L_{3} F_{3} S_{3} J_{3} J_{z 3}>$. The two-photon transitions are described with the two-photon transition operator $Q_{\varepsilon_{1} \varepsilon_{2}}^{S}$. The second-order time dependent perturbation theory allows to calculate the twophoton transition rates, expressed as :

$$
\Gamma_{\mathrm{ge}}^{(2)}=\frac{P_{1} P_{2}}{\left(S c \hbar \varepsilon_{0}\right)^{2}}\left|\left\langle g\left|Q_{p_{1} p_{2}}^{S}\right| e\right\rangle\right|^{2} \frac{\Gamma_{\text {eff }}}{4 \delta \omega^{2}+\Gamma_{\text {eff }}^{2} / 4}
$$

in function of the lifetimes of the excited $\gamma_{e}$ and ground state $\gamma_{g}$, where $\Gamma_{\text {eff }}=\left(\gamma_{e}+\gamma_{g}\right) / 2$, the detuning of the two-photon transition $\delta \omega=\omega-\omega_{\mathrm{ge}} / 2$, the level splitting $\hbar \omega_{g e}=E_{e}-E_{g}$, the reduced Planck constant $\hbar$, the electric constant $\varepsilon_{0}$ and the speed of light $c$. The two-photon operator is expanded with the scalar and quadrupolar spherical tensors :

$$
Q_{p_{1} p_{2}}^{S}=\sum_{p=-2}^{2}\left\langle 11 p_{1} p_{2} \mid 2 p\right\rangle Q_{p}^{(2)}+\left\langle 11 p_{1} p_{2} \mid 00\right\rangle Q_{0}^{(0)}
$$


The matrix elements of the two-photon operator between two pure magnetic states are expressed as :

$$
\begin{gathered}
\left\langle g\left|Q_{p}^{(k)}\right| e\right\rangle=(-1)^{J_{1}-J_{z 1}}\left(\begin{array}{ccc}
J_{1} & k & J_{2} \\
-J_{z 1} & p & J_{z 2}
\end{array}\right)\left\langle v_{1} L_{1} F_{1} S_{1} J_{1}\left\|Q^{(k)}\right\| v_{2} L_{2} F_{2} S_{2} J_{2}\right\rangle \\
\left\langle v_{1} L_{1} F_{1} S_{1} J_{1}\left\|Q^{(k)}\right\| v_{2} L_{2} F_{2} S_{2} J_{2}\right\rangle=\sqrt{2 k+1} \sum_{r} R e\left[\begin{array}{c}
\frac{\left\langle v_{1} L_{1} F_{1} S_{1} J_{1}\|D\| v_{r} L_{r} F_{r} S_{r} J_{r}\right\rangle\left\langle v_{r} L_{r} F_{r} S_{r} J_{r}\|D\| v_{2} L_{2} F_{2} S_{2} J_{2}\right\rangle}{\hbar\left(\omega-\omega_{g r}+i\left(\gamma_{r}+\gamma_{g}\right) / 2\right)} \\
\times\left\{\begin{array}{ccc}
J_{1} & J_{2} & k \\
1 & 1 & J_{r}
\end{array}\right\}(-1)^{J_{1}+J_{2}+k}
\end{array}\right. \\
\left\langle v_{1} L_{1} F_{1} S_{1} J_{1}\|D\| v_{2} L_{2} F_{2} S_{2} J_{2}\right\rangle=\delta_{S_{1} S_{2}(-1)^{2 L_{1}+S_{1}+J_{2}+1} \sqrt{\left(2 J_{1}+1\right)\left(2 J_{2}+1\right)\left(2 L_{1}+1\right)\left(2 L_{2}+1\right)}} \\
\times\left\{\begin{array}{ccc}
L_{2} & J_{2} & S_{1} \\
J_{1} & L_{1} & 1
\end{array}\right\}\left(\begin{array}{ccc}
L_{1} & 1 & L_{2} \\
0 & 0 & 0
\end{array}\right) \cdot \mu_{v_{1} L_{1}, v_{2} L_{2}}
\end{gathered}
$$

using the Wigner-Eckart theorem, in function of the detuning to the intermediary level $\omega-\omega_{\mathrm{gr}}$, the level splitting $\hbar \omega_{g r}=E_{r}-E_{g}$, the lifetime of the relay state $\gamma_{r}$, and the contributions from the matrix elements of the electric dipole operator rotated in the space-fixed system $D$, that are expressed on the last lines in function of the transition dipole moments calculated in ref. ${ }^{20}$.

\section{A MOLECULAR ION CLOCK BASED ON TWO-PHOTON TRANSITIONS OF HD ${ }^{+}$}

This contribution proposes to exploit a molecular ion clock based on the magnetic components of the two-photon transitions $(v, L)=(0,0) \rightarrow(2,0)$ or $(v, L)=(0,0) \rightarrow(2,2)$ of the $\mathrm{HD}^{+}$ions. The experimental setup (Fig. 1.a), similar to that used in previous approaches ${ }^{14,15}$, is based on an ion trap that store $\sim 10^{2} \mathrm{HD}^{+}$ions sympathetically cooled with $\sim 10^{3}$ co-trapped $\mathrm{Be}^{+}$ions that are laser-cooled at $313 \mathrm{~nm}$. The detection of the two-photon transitions is performed by photodissociating with a $175 \mathrm{~nm}$ laser the $\mathrm{HD}^{+}$ions transferred to the $v=2$ level (Fig. 1.b). The number of $\mathrm{HD}^{+}$ions is determined by monitoring the fluorescence of $\mathrm{Be}^{+}$ions when the secular motion of the $\mathrm{HD}^{+}$ions is excited in the trap. An uniform static magnetic field is generated with three coil pairs in the Helmholtz configuration which are driven with three independent current sources and applied to the ion trap.

The $\mathrm{HD}^{+}$ion population is distributed initially among the rotational levels of the ground vibrational state. The blackbody radiation drives electric dipole allowed transitions between these rotational levels. A stationary wave from a laser at $55.909 \mathrm{THz}$, or $57.701 \mathrm{THz}$, respectively, which is referenced to a frequency standard, drives the two-photon transition. An efficient frequency stabilization scheme is required to provide ultrastable laser radiation in order to probe Doppler-free infrared lines of $\mathrm{HD}^{+}$at the limit of their radiative linewith. 
The feasibility of the detection scheme and the metrological performances from degenerate twophoton infrared spectroscopy were discussed in ref. ${ }^{21}$. The lineshape of the signal was calculated by solving a coupled system of rate equations for the populations in the relevant rovibrational levels of $\mathrm{HD}^{+}$(the hyperfine structure is neglected), by taking into account the two-photon transition, the dissociation, the radiative relaxation, and the transitions driven by the blackbody radiation. The twophoton peak is power broadened, with a full width at half-maximum of $\sim 20 \mathrm{~Hz}$ for a two photon transition rate of $10 \mathrm{~s}^{-1}$ and a dissociation rate of $200 \mathrm{~s}^{-1}$. The lightshift is at the Hz level.

A determinant metrological characteristic is the stability of the molecular ion clock, quantified with the Allan variance, that is associated to the uncertainty in absolute frequency measurements of the $\mathrm{HD}^{+}$transitions. This uncertainty is translated directly, through the sensitivity of the molecular ion transitions to the external fields, to the uncertainty of the spectroscopic calibration of the external fields. In the case of the single ion clocks the uncertainty is dominated by the quantum projection noise that scales inversely proportional to the squared root of the interrogation time and to the quality factor of the molecular ion transition ${ }^{22}$. In the case of measurements limited by the radiative linewidth of the infrared transitions, the frequency uncertainty for the $(v, L)=(0,0) \rightarrow(2,0)$ line is estimated at $2.49 \mathrm{~Hz}$ and for the $(v, L)=(0,0) \rightarrow(2,2)$ line at $2.57 \mathrm{~Hz}$.

An important feature is an efficient quantum state preparation of $\mathrm{HD}^{+}$in the lower energy level. The ground rotational state $(v, L)=(0,0)$ of $\mathrm{HD}^{+}$is interesting because of its simpler hyperfine structure. The enhancement of the population in the $(v, L)=(0,0)$ level was demonstrated with the rotational cooling scheme ${ }^{23}$. In addition, hyperfine state preparation may be performed with the approach proposed in ref. ${ }^{24}$, by associating the rotational cooling with the optical pumping of selected hyperfine levels with state-selective two-photon transitions.

The stretched states are the magnetic states with maximum angular momentum $J$ and absolute projection $J_{z}$. The transitions between the stretched states $\left(v, L, F=1, S=2, J=L+2, J_{z}= \pm(L+2)\right) \rightarrow\left(v^{\prime}\right.$, $\left.L^{\prime}, F^{\prime}=1, S^{\prime}=2, J^{\prime}=L^{\prime}+2, J_{z}^{\prime}= \pm\left(L^{\prime}+2\right)\right)$ of $\mathrm{HD}^{+}$are metrologically interesting because they are affected by a linear Zeeman shift, that open the possibility of cancellation by exploiting the mean frequency. In addition, the quadrupole shift is equal for the two states and independent on the magnetic field ${ }^{9}$. The quadrupole shift is null for the $L=0$ states $^{9}$. For other states the quadrupole shift may be cancelled by averaging the transitions measured for three orthogonal directions of the magnetic 
field $^{25}$. The two-photon clock transitions addressed here start from the $L=0$ level with the advantage of simple hyperfine structure that open the possibility for hyperfine state preparation :

- transition (1) between the stretched states $\left(v, L, F, S, J, J_{z}\right)=(0,0,1,2,2,2) \rightarrow(2,0,1,2,2,2)$ is used for probing the lightshifts induced by the THz-wave. The advantages of this transition are suppressed quadrupole shift and the very low sensitivity to the magnetic field.

- transition (2) $\left(v, L, F, S, J, J_{z}\right)=(0,0,1,2,2,-2) \rightarrow(2,2,1,2,4,0)$ is used to characterize by spectroscopy the magnetic field. The advantages of this transition are high two-photon transition rate ${ }^{26}$ and sensitivity to the magnetic field.

The metrological performances of the two-photon $\mathrm{HD}^{+}$infrared clock are indicated in Table 1. The hypotheses and methods to calculate the systematic shifts and their uncertainties are indicated below

1) Zeeman shift. It is the dominant systematic shift and therefore a small static magnetic field of $2 \mu \mathrm{T}$ is assumed for clock operation. The energy levels shifts may be expressed with a quadratic dependence in function of the magnetic field ${ }^{7}$, for small to moderate $\left(10^{-4} \mathrm{~T}\right)$ fields. In this case for the transition (1), the theoretical linear Zeeman coefficient is negligible $\left(<10^{4}\right.$ $\mathrm{Hz} / \mathrm{T}$ ) and the uncertainty is assumed conservatively as the value of the theoretical error for the nominal magnetic field value, that is $0.02 \mathrm{~Hz}$. The frequency of the transition (2) is expressed approximately as the sum of a relatively important first order Zeeman shift and a small second-order Zeeman shift $f=f_{0}+\eta_{B} B+\eta_{B^{2}} B^{2}$. In this case it is interesting to measure also the transition $\left(v, L, F, S, J, J_{z}\right)=(0,0,1,2,2,2) \rightarrow(2,2,1,2,4,0)$ that depends approximately on the magnetic field as $f^{\prime}=f_{0}-\eta_{B} B+\eta_{B^{2}} B^{2}$. In this case the mean frequency of these transitions is affected by a small shift depending quadratically on the magnetic field. Careful measurements of the mean frequency in function of the magnetic field may allow to determine the second-order Zeeman shift with an uncertainty of $0.2 \%$ of its value, that is approximately $0.032 \mathrm{~Hz}$ for the $2 \mu \mathrm{T}$ magnetic field. In addition, careful measurements of the splitting between these two transitions in function of the magnetic field may allow to determine the linear part in the Zeeman shift with an uncertainty of $14 \mathrm{~Hz}$, that is $0.1 \%$ of the shift value. 
2) Trap shift. Several effects, such as micromotion induced Stark shift, phase-offset induced Stark shift ${ }^{27}$, lead to a dependence proportional with the mean square value of the electric field in the ion trap. They were measured accurately for components of the transition $(v, L)=(0,0) \rightarrow(0,1)$ of $\mathrm{HD}^{+}$in ref. ${ }^{14}$. For this experimental setup, the transition between the stretched states, recorded with $4 \mathrm{~Hz}$ FWHM linewidth, is shifted by $-6 \mathrm{~Hz}$ when decreasing the trap electric field amplitude by $2.5 \mathrm{~V}$. Rescaling of this result to the case of the twophoton infrared transitions of $\mathrm{HD}^{+}$is performed here by using the static scalar polarizabilities of the hyperfine levels of $\mathrm{HD}^{+}$calculated in ref. ${ }^{8}$ and by assuming that the electric field is parallel with the quantization axis. The trap shift for the transition (1) is $-45 \mathrm{~Hz}$ and for the transition (2) is $0.58 \mathrm{~Hz}$. The uncertainty is dominated by the error of the trap shift coefficient derived from the rotational spectroscopy measurement with an assumed uncertainty of $(4 \mathrm{~Hz}) \times \sqrt{2} / 10$, that lead to a fractional uncertainty of $9 \%$. The ac-Zeeman effect may also contribute to the trap shift but it was not observed experimentally ${ }^{14}$.

3) Second order Doppler shift. It arises from the micromotion of the molecular ion and is related to the Stark shift experienced in the ion trap. As indicated in ref. ${ }^{27}$, the ratio of the second order Doppler shift to the Stark shift may be expressed as :

$$
\frac{\Delta f_{D 2}}{\Delta f_{S}}=\frac{h f}{\Delta \alpha_{s}}\left(\frac{e}{m \Omega c}\right)^{2}
$$

in function of the trap frequency $\Omega / 2 \pi$, the electric charge $e$, the $\mathrm{HD}^{+}$ion mass $m$, the transition energy $h f$ and differential polarizability $\Delta \alpha_{s}$. Using the static scalar polarizabilities from ref. ${ }^{8}$ and a trap frequency of $\Omega / 2 \pi=14.16 \mathrm{MHz}$, the value of the shift is $-2.0 \mathrm{~Hz}$ for transition (1), and $-0.073 \mathrm{~Hz}$ for transition (2). The uncertainties are assumed fractionally at $9 \%$ as for the trap shift.

4) Blackbody radiation shift. It was calculated for electric dipole coupling of the blackbody radiation field (BBR) at room temperature $T=300 \mathrm{~K}$ to the rovibrational levels of $\mathrm{HD}^{+}$in ref. $^{8}$. The shift is assumed the same for the hyperfine states of a given rovibrational level. The effect of the BBR magnetic field is neglected. The estimations using the dominant contributions from static and dynamic rovibrational polarizabilities give a BBR shift of - 
3.3(3) $\mathrm{mHz}$ for transition (1) and $-7.8(4) \mathrm{mHz}$ for transition (2). The uncertainties indicated in parentheses are calculated by assuming the temperature uncertainty of $5 \mathrm{~K}$.

5) Quadrupole shift. It was calculated at the first order perturbation theory using an effective Hamiltonian in function of the quadrupole coefficients of the $\mathrm{HD}^{+}$rovibrational energy levels in ref. ${ }^{9}$. The quadrupole shifts for the $L=0$ states of $\mathrm{HD}^{+}$are zero, therefore the transition (1) has no quadrupole shift. The quadrupole shift for the transition (2) is estimated at $-1.7 \mathrm{~Hz}$ for an electric field gradient $V_{z z}=67 \mathrm{MV} / \mathrm{m}^{2}$. Cancellation of the quadrupole shift may be performed by taking the mean of the frequencies of the transition measured for three orthogonal directions of the magnetic field by keeping the same value of the magnetic field. The uncertainty coming from the averaging procedure for 10 independent sets of measurements is $0.47 \mathrm{~Hz}$. Establishing correctly the three orthogonal directions for the magnetic field is an another source of uncertainty, that was evaluated fractionally at less than $5 \times 10^{-17}$ for the mercury ion clock $^{28}$, that rescales in the case of the infrared $\mathrm{HD}^{+}$clock to a fractional value better than $5 \times 10^{-16}$, as it is estimated in ref. ${ }^{9}$. The uncertainty in the cancellation of the quadrupole shift is dominated by the averaging procedure.

6) Lightshift due to the spectroscopy laser. The value is $-1.09 \mathrm{~Hz}$ for transition (1) at the nominal intensity of $38.7 \mathrm{~W} / \mathrm{cm}^{2}$, as calculated in ref. ${ }^{21}$ using the two-photon operator formalism. The value of the intensity that ensures the same two-photon transition rate for transition (2) is estimated at $7.04 \mathrm{~W} / \mathrm{cm}^{2}$ by using effective two-photon transition matrix elements from ref. ${ }^{26}$ and the energy level lifetimes for the $\mathrm{HD}^{+}$ion ${ }^{19}$. The value of the lightshift for transition (2) is estimated at $0.56 \mathrm{~Hz}$. The uncertainties are assumed at $1 \%$ of the lightshift values.

7) Lightshift due to the cooling laser. It is estimated in ref. ${ }^{21}$ with the extrapolated values of the scalar polarizabilities of the $\mathrm{HD}^{+}$energy levels from ref. ${ }^{8}$ for a nominal intensity of 0.8 $\mathrm{mW} / \mathrm{mm}^{2}$ of the $313 \mathrm{~nm}$ laser. The value of the lighshift is $-1.3 \mathrm{mHz}$ for both transitions. The uncertainties are assumed at $1 \%$ of the lightshift values.

8) Lightshift due to the dissociating laser. It is estimated in ref. ${ }^{21}$ with extrapolated values of the scalar polarizabilities of the $\mathrm{HD}^{+}$energy levels calculated in ref. ${ }^{8}$ for a nominal intensity 
of $6.1 \mathrm{~W} / \mathrm{cm}^{2}$ of the $175 \mathrm{~nm}$ laser. The value of the lighshift is $-0.1 \mathrm{~Hz}$ for both transitions. The uncertainties are assumed at $1 \%$ of the lightshift values.

Examination of Table 1 allows to derive the fractional accuracy of $7.3 \times 10^{-14}$ for the transition (1) and $2.4 \times 10^{-13}$ for the transition (2), respectively. The precision is limited by the trap shift for transition (1), and by the relatively important Zeeman shift that is required for magnetic field sensing in the case of the transition (2). The reduction of the Stark and Zeeman effects and the exploitation of composite frequencies to cancel effectively the systematic effects ${ }^{29}$ may open perspectives for infrared clocks with cold trapped $\mathrm{HD}^{+}$ions having fractional accuracies at the $10^{-15}$ level. The uncertainty of the frequency measurements with these clocks will be limited by the molecular ion quantum projection noise as quoted previously.

\section{MEASURING A MAGNETIC FIELD BY ZEEMAN SPECTROSCOPY OF HD ${ }^{+}$}

Zeeman spectroscopy of the transition (2) is exploited here to calibrate the strength of the static magnetic field in the ion trap up to values by $10^{-4} \mathrm{~T}$. The Zeeman shift is calculated approximately with a quadratic dependence:

$$
\delta f_{z}^{(2)}(\mathrm{B})=f^{(2)}(\mathrm{B})-f^{(2)}(\mathrm{B}=0)=\eta_{B}\left(\left\{V_{t h}\right\}\right) B+\eta_{B^{2}}\left(\left\{V_{t h}\right\}\right) B^{2}
$$

in function of the magnetic field strength $B$, the linear $\eta_{B}$ and quadratic $\eta_{B^{2}}$ Zeeman shift coefficients that are expressed in function of six parameters $\left\{V_{t h}\right\}=\left\{t_{1}, q_{1}, r_{1}, t_{2}, q_{2}, r_{2}\right\}$ calculated abinitio for the $|1>=| 0,0,1,2,2,-2>$ and $|2>=| 2,2,1,2,4,0>$ hyperfine states of $\mathrm{HD}^{+}$in ref. ${ }^{7}$. Inversing this equation yields the magnetic field strength in function of the experimental Zeeman shift and the values for the theoretical parameters $\left\{U_{t h}\right\}$. In the case of two possible solutions, the magnetic field strength value is chosen to match the result of an independent measurement with an accurate magnetometer. The uncertainty of the spectroscopic measurement is derived by propagating the errors from the Zeeman shift frequency measurement $u\left(\delta f_{z}^{(2)}\right)=2.57 \cdot \sqrt{2} \mathrm{~Hz}$ and from the Zeeman shift parameters $u\left(q_{1,2}\right)=u\left(r_{1,2}\right)=50 \mathrm{MHz} / \mathrm{T}^{2}, u\left(t_{1,2}\right)=5 \mathrm{kHz} / \mathrm{T}$. The total uncertainty of the magnetic field measurement in function of the value of the magnetic field is calculated and plotted in Fig. 2, as well as the partial contribution from theory and experiment errors. The accuracy of the calibration of the magnetic field by spectroscopy $\sim 5 \times 10^{-10} \mathrm{~T}$ is limited by frequency measurement error in this range of magnetic field values. The contribution of the theoretical errors to the total magnetic field 
uncertainty is significantly smaller. Particularly, the quadratic dependence of the Zeeman shift in function of the magnetic field assumed here allows to reach a minimal theoretical uncertainty by 2 fT for a magnetic field of $4.99 \times 10^{-5} \mathrm{~T}$.

\section{LIGHSHIFTS OF TWO-PHOTON TRANSITIONS OF HD ${ }^{+}$FOR THZ ELECTROMETRY}

\subsection{Determination of the amplitude of a $\mathrm{THz}$ electric field}

The measurements of the lightshifts induced by a THz-wave on the transition (1) are exploited here to calibrate the electric field of a THz-wave. The lightshift may be expressed in function of the $\mathrm{THz}$ electric field amplitude $\mathrm{E}_{\mathrm{THz}}$ and the difference $\Delta \alpha\left(\left\{U_{t h}\right\} ; B, f_{T H z}\right)$ of the total dynamic polarizabilities of the states $|1>=| 0,0,1,2,2,2>$ and $|2>=| 2,0,1,2,2,2>$, which is calculated in function of a set of theoretical parameters $\left\{U_{t h}\right\}$, the strength of the magnetic field $B$ and the frequency of the THz-wave $f_{T H z}:$

$$
\delta f_{L S}^{(1)}\left(E_{T H z}\right)=f^{(1)}\left(E_{T H z}\right)-f^{(1)}\left(E_{T H z}=0\right)=-\frac{E_{T H z}^{2}}{8}\left(\alpha_{2}\left(\left\{U_{t h}\right\} ; B, f_{T H z}\right)-\alpha_{1}\left(\left\{U_{t h}\right\} ; B, f_{T H z}\right)\right)
$$

The uncertainty of the $\mathrm{THz}$ electric field is calculated by propagating the THz-wave frequency uncertainty, the frequency uncertainties of the $\mathrm{HD}^{+}$transitions, that contribute to the uncertainties of the lightshift and of the calibrated magnetic field strength, and the uncertainties of the theoretical parameters, that contribute to the uncertainties of the calculated dynamic polarizabilities and of the magnetic field strength. The dependence is expressed as :

$$
u\left(E_{T H z}\right)=\sqrt{\frac{2 u^{2}\left(\delta f_{L S}^{(1)}\right)}{\left(-\delta f_{L S}^{(1)} \cdot \Delta \alpha\right.}+\frac{E_{T H z}^{2} \cdot\left(\frac{\cdot u(\Delta \alpha)}{\Delta \alpha}\right)^{2}}{2}}
$$

in function of the uncertainty of the lightshift measurement $u\left(\delta f_{L S}^{(1)}\right)$, and the uncertainty of the difference of dynamic polarizabilities $u\left(\Delta \alpha\left(\left\{U_{t h}\right\} ; B, f_{T H z}\right)\right)$. The lowest THz electric field that may be detected is associated to the smallest lightshift that can be observed, that is $u\left(\delta \delta_{L S}^{(1)}\right)=2.49 \cdot \sqrt{2} \mathrm{~Hz}$. The value is estimated numerically as $u\left(E_{T H z}\right)=u\left(\delta f_{L S}^{(1)}\right) \sqrt{-2 /\left(\delta f_{L S}^{(1)} \cdot \Delta \alpha\right)}$. The fractional uncertainty for high $\mathrm{THz}$ electric fields scales as the fractional uncertainty of the difference of dynamic polarizabilities. Changing the strength of the magnetic field in the ion trap may be used to tune dipole-allowed 
transitions starting from $\left(v, L, F, S, J, J_{z}\right)=(0,0,1,2,2,2)$ to sublevels of $(v, L)=(0,1)$ near the frequency of the THz-wave. Near each resonance, the dynamic polarizability $\alpha_{1}\left(\left\{U_{t h}\right\} ; B, f_{T H z}\right)$ displays a dispersive dependence and its uncertainty increases significantly. Decreasing the detuning may enhance the sensitivity but the precision may become smaller. An optimal value of the magnetic field has to be chosen to minimize the uncertainty given by eq. (10).

Let us consider the case of the measurements of the lightshifts of the $\left(v, L, F, S, J, J_{z}\right)=(0,0,1,2,2,2) \rightarrow(2,0,1,2,2,2) \quad$ two-photon transition (experimental uncertainty $u\left(\delta f_{L S}\right)=2.49 \cdot \sqrt{2} \mathrm{~Hz}$ ) to detect a THz-wave (frequency $1,314,947,502.3 \mathrm{kHz}, \pi$ polarization) that is slightly detuned to the hyperfine component of the rotational transition $(v, L, F, S, J)=(0,0,1,2,2) \rightarrow(0,1,1,2,3)$. The electric dipole couplings are on the $\left(v, L, F, S, J, J_{z}\right)=(0,0,1,2,2,2) \rightarrow(0,1,1,2,3,2)$ and $\left(v, L, F, S, J, J_{z}\right)=(0,0,1,2,2,2) \rightarrow(0,1,1,2,2,2)$ transitions. The optimization procedure for the magnetic field strength yields the value $6 \mu \mathrm{V} / \mathrm{m}$ for the weakest electric field that may be detected with this method. This is a 30 times improvement comparing to the limit of microwave electric field detection by Rydberg atom spectroscopy in vapour cell ${ }^{4}$.

Then, the value of the magnetic field that minimizes the total uncertainty in eq. (10) is optimized for different values of the $\mathrm{THz}$ electric field amplitude. That yields the dependence of the accuracy of the THz-wave electric field calibration that is plotted in Fig. 3. The accuracy of the THz electric field calibration is by tens of $\mu \mathrm{V} / \mathrm{m}$ that is limited by the accuracy of the dynamic polarizability calculation. The contribution to the uncertainty arising from the frequency measurement errors is by an order of magnitude smaller than the total uncertainty.

\subsection{Three-dimensional characterization of a THz electric field}

The THz electric field vector is described in the Cartesian laboratory coordinate frame $L C F\left(\vec{e}_{x}, \vec{e}_{y}, \vec{e}_{z}\right)$

(Fig. 1.a.) with three orthogonal linearly polarized components oscillating along each axis:

$$
\vec{E}(\mathrm{t})=\sum_{j=\{x, y, z\}} \frac{E_{j} \vec{e}_{j}}{2} \times \mathrm{e}^{-i\left(\omega t+\varphi_{j}\right)}+\text { c.c. }
$$

The THz electric field vector is characterized with three real positive amplitudes $E_{x}, E_{y}, E_{z}$ and two relative phases $\varphi_{x}, \varphi_{y}$, the other being fixed to zero $\varphi_{z}=0$. The set of $\left(E_{x}, E_{y}, E_{z}, \varphi_{x}, \varphi_{y}\right)$ parameters may 
be related to the squared amplitudes of the $\mathrm{THz}$ electric field standard components $\left|E_{-1,0,1}^{(\alpha, \beta)}\right|^{2}$ in a Cartesian frame rotated in function of the direction of the quantization axis with Euler angles $(\alpha, \beta, \chi=0)^{30,31}$ (Fig. 1.a.). The lightshift of the two-photon transition is expressed in eq. (3) with the standard components of the $\mathrm{THz}$ electric field. The inversion of this approach may provide the amplitudes and the phases of the Cartesian components of the $\mathrm{THz}$ electric field from the lightshift measurements.

Six lightshift measurements $F_{L S}=\left\{\delta f_{L S}^{(0,0)}\left(B_{1}\right), \delta f_{L S}^{(0,0)}\left(B_{2}\right), \delta f_{L S}^{(0,0)}\left(B_{3}\right), \delta f_{L S}^{(0, \pi / 2)}\left(B_{1}\right), \delta f_{L S}^{(0, \pi / 2)}\left(B_{2}\right), \delta f_{L S}^{(0, \pi / 2)}\left(B_{3}\right)\right\}$ may be performed for the two-photon transition $\left(v, L, F, S, J, J_{z}\right)=(0,0,1,2,2,2) \rightarrow(2,0,1,2,2,2)$ using two orientations of the magnetic field $(\alpha, \beta)=(0,0),(\alpha, \beta)=(0, \pi / 2)$ and three different magnetic field strengths $B_{1}=10^{-6} \mathrm{~T}, B_{2}=5 \times 10^{-6} \mathrm{~T}, B_{3}=10^{-5} \mathrm{~T}$. The lightshifts are related to the squared amplitudes of the standard components of the THz electric field $E_{S T D}^{2}=\left\{\left|E_{-1}^{(0,0)}\right|^{2},\left|E_{0}^{(0,0)}\right|^{2},\left|E_{1}^{(0,0)}\right|^{2},\left|E_{-1}^{(0, \pi / 2)}\right|^{2},\left|E_{0}^{(0, \pi / 2)}\right|^{2},\left|E_{1}^{(0, \pi / 2)}\right|^{2}\right\}$ with a $6 \times 6$ matrix $A\left(\Delta \alpha_{i, q}\left(\left\{U_{t h}\right\} ; B, f_{T H z}\right)\right)$ formed with two $3 \times 3$ diagonal blocks expressed in function of differential standard dynamic polarizabilities of the two-photon transitions. If the matrix is nonsingular, the squared amplitudes of the standard components may be determined by inversion $E_{S T D}^{2}=\left[A\left(\Delta \alpha_{i, q}\right)\right]^{-1} \cdot F_{L S}$. Nonsingularity may be satisfied by using three different magnetic field strengths. Finally, the parameters of the THz-wave in the LCF may be expressed with analytical dependences in function of $\left(\left|E_{-1}^{(0,0)}\right|^{2},\left|E_{0}^{(0,0)}\right|^{2},\left|E_{1}^{(0,0)}\right|^{2},\left|E_{-1}^{(0, \pi / 2)}\right|^{2},\left|E_{1}^{(0, \pi / 2)}\right|^{2}\right)$ :

$$
\begin{aligned}
& E_{x}=\sqrt{\left|E_{1}^{(0,0)}\right|^{2}+\left|E_{-1}^{(0,0)}\right|^{2}-\left(\left|E_{1}^{(0, \pi / 2)}\right|^{2}+\left|E_{-1}^{(0, \pi / 2)}\right|^{2}-\left|E_{0}^{(0,0)}\right|^{2}\right)} \\
& E_{y}=\sqrt{\left|E_{1}^{(0, \pi / 2)}\right|^{2}+\left|E_{-1}^{(0, \pi / 2)}\right|^{2}-\left|E_{0}^{(0,0)}\right|^{2}} \\
& E_{z}=\left|E_{0}^{(0,0)}\right| \\
& \varphi_{x}=\varphi_{y}+\arcsin \left(\frac{\left|E_{1}^{(0,0)}\right|^{2}-\left|E_{-1}^{(0,0)}\right|^{2}}{2 E_{x} E_{y}}\right) \\
& \varphi_{y}=\arcsin \left(\frac{\left|E_{1}^{(0, \pi / 2)}\right|^{2}-\left|E_{-1}^{(0, \pi / 2)}\right|^{2}}{2 E_{y} E_{z}}\right)
\end{aligned}
$$


Let us discuss the application of this method for the characterization of a reference THz-wave at frequency $f_{T H z}=1,314,947,502.3 \mathrm{kHz}$ that is linearly polarized along an arbitrary direction defined in the $L C F$ with Euler angles $\phi$ and $\theta$ (Fig. 1.a). The amplitudes of the THz electric field may be expressed as $\left(E_{\mathrm{x}}, E_{\mathrm{y}}, E_{\mathrm{z}}\right)=\left(E_{0} \cdot \sin \theta \cdot \cos \phi, E_{0} \cdot \sin \theta \cdot \sin \phi, E_{0} \cdot \cos \theta\right)$ in function of the amplitude of the THzwave $E_{0}$. The phases are assumed as $\varphi_{\mathrm{x}}=\varphi_{\mathrm{y}}=\varphi_{\mathrm{z}}=0$. The performances are evaluated using a Monte Carlo calculation approach. For the reference THz-wave with a given frequency, intensity and polarization orientation, the experimental lightshift is first estimated by adding to the molecular ion theory prediction a random error described with a Gaussian distribution with zero mean and a standard deviation of $u\left(\delta f_{L S}\right)=2.49 \cdot \sqrt{2} \mathrm{~Hz}$. Then, the squared amplitudes of the standard components of the $\mathrm{THz}$ electric field are derived using the relevant differential standard dynamic polarizabilities. Each polarizability is estimated as the theory value plus a random error with a Gaussian distribution with zero mean and standard deviation corresponding to the polarizability calculation uncertainty. Finally, the parameters of the THz electric field in the laboratory frame are determined with eq. (12).

The mean values and uncertainties for the amplitudes and phases of the Cartesian components of the electric field vector are derived from 10 sets of simulations for a THz-wave with an intensity of 1 $\mathrm{W} / \mathrm{m}^{2}$ (electric field amplitude of $E_{0}=27.42 \mathrm{mV} / \mathrm{m}$ ). The uncertainties are plotted in Fig. 4 in function of the spherical angle parameterization for the polarization orientation. The uncertainties for $E_{\mathrm{x}}, E_{\mathrm{y}}$ are at the $\mathrm{mV} / \mathrm{m}$ level or better, for $E_{\mathrm{z}}$ are at the $0.1 \mathrm{mV} / \mathrm{m}$ level or better, and for $\varphi_{\mathrm{x}}, \varphi_{\mathrm{y}}$ are in general better than $0.1 \mathrm{rad}$. In the case of very small values of the amplitude of the $\mathrm{THz}$ electric field, that arise for specific orientations of the polarization vector, the deviation between the mean value and the reference value of the amplitude is significantly higher than the uncertainty.

In addition, for a specific orientation of the polarization vector $(\theta=\pi / 4, \phi=\pi / 4)$, the parameters of the THz-wave are calculated for different amplitudes of the $\mathrm{THz}$ electric field (corresponding to intensities from $0.1 \mathrm{~W} / \mathrm{m}^{2}$ to $10 \mathrm{~W} / \mathrm{m}^{2}$ ). The uncertainties of the parameters, plotted in Fig. 5, reduce with the increase of the amplitude of the $\mathrm{THz}$ electric field. The deviation between the mean value obtained by measurement for each parameter of the THz-wave and the reference value is compatible with the uncertainty of the measurement. In the case of a THz-wave with an intensity of $10 \mathrm{~W} / \mathrm{m}^{2}$, this method may allow to calibrate the amplitudes of the $\mathrm{THz}$ electric field with $100 \mu \mathrm{V} / \mathrm{m}$ level accuracy or better, and the phases with mrad level accuracy. 


\section{CONCLUSION}

This contribution proposes a new approach to characterize the electromagnetic fields by comparing the measurements of the frequency shifts induced on $\mathrm{HD}^{+}$clock transitions with the predictions of the molecular ion theory. The degenerate two-photon spectroscopy of cold trapped $\mathrm{HD}^{+}$ions may provide $10^{-14}$ accuracy or better in the infrared region. The frequencies of the $\mathrm{HD}^{+}$transitions and their frequency shifts in external fields may be calculated with precision using the quantum theory. This method enables therefore a direct link of the external fields to the SI second and to a set of fundamental constants provided by CODATA2018 ${ }^{32}$. The uncertainties of the parameters of the electromagnetic fields may be derived from the uncertainties of the frequency measurements of $\mathrm{HD}^{+}$ transitions and the uncertainties of the theoretical calculations.

Precisely, the systematic frequency shifts of the molecular ion clocks based on the two-photon transitions $\left(v, L, F, S, J, J_{z}\right)=(0,0,1,2,2,-2) \rightarrow(2,2,1,2,4,0)$ and $\left(v, L, F, S, J, J_{z}\right)=(0,0,1,2,2,2) \rightarrow(2,0,1,2,2,2)$ of $\mathrm{HD}^{+}$may be exploited for the characterization of the electromagnetic fields. The systematic frequency shifts are accurately estimated and the uncertainty of the measurements of the frequency shifts is assumed at the limit from the quantum projection noise in single-ion experiments. The Zeeman shift of the first clock transition may be exploited to measure the strength of the static magnetic field applied to the ion trap with an uncertainty at the $10^{-10} \mathrm{~T}$ level. The ligthtshift of the second clock transition may be exploited to characterize the electric field of a THz-wave tuned near the hyperfine transition $(v, L, F, S, J)=(0,0,1,2,2) \rightarrow(0,1,1,2,3)$ of $\mathrm{HD}^{+}$. The lowest electric field that may be detected is at the $\mu \mathrm{V} / \mathrm{m}$ level and the accuracy of the calibration is at the $10 \mu \mathrm{V} / \mathrm{m}$ level. The electric field of the THz-wave may be characterized in three dimensions using six lightshift measurements for two orientations of the magnetic field that defines the $\mathrm{HD}^{+}$quantization axis and three values of its strength. A THz-wave with linear polarization and an intensity of $10 \mathrm{~W} / \mathrm{m}^{2}$ may be calibrated using ten sets of lightshift measurements with an accuracy estimated at $0.1 \mathrm{mV} / \mathrm{m}$ level or better for the amplitudes of the Cartesian components of the THz electric field, and an accuracy at the mrad level for the phases. 


\section{REFERENCES}

[1] Haroche, S., "Nobel Lecture: Controlling photons in a box and exploring the quantum to classical boundary,“ Rev. Mod. Phys. 85 (3), 1083 (2013).

[2] Facon, A., Dietsche, E.-K., Grosso, D., Haroche, S., Raimond, J.-M., Brune, M. and Gleyzes, S., "A sensitive electrometer based on a Rydberg atom in a Schrödinger-cat state," Nature 535, 262 265 (2016).

[3] Sedlacek, J.A., et al, "Microwave electrometry with Rydberg atoms in a vapour cell using bright atomic resonances," Nat. Phys. 8, 819-824 (2012).

[4] Kumar, S., et al, "Rydberg-atom based radio-frequency electrometry using frequency modulation spectroscopy in room temperature vapour cells," Opt. Express 25 (8), 8625-8637 (2017).

[5] Korobov, V.I., Hilico, L. and Karr, J.-Ph., "Fundamental transitions and ionization energies of the hydrogen molecular ions with few ppt uncertainty," Phys. Rev. Lett. 118 (23), 233001 (2017).

[6] Korobov, V.I., Karr, J.-Ph., Haidar, M. and Zhong, Z.-X., "Hyperfine structure in the $\mathrm{H}_{2}^{+}$and $\mathrm{HD}^{+}$molecular ions at order ma $\alpha^{6}$," Phys. Rev. A 102 (2), 022804 (2020).

[7] Bakalov, D., Korobov, V.I. and Schiller, S., "Magnetic field effects in the transitions of the HD" molecular ion and precision spectroscopy,” J. Phys. B 44 (2), 025003 (2011).

[8] Schiller, S., Bakalov, D., Bekbaev, A.K. and Korobov, V.I., "Static and dynamic polarizability and the Stark and blackbody-radiation frequency shifts of the molecular hydrogen ions $\mathrm{H}_{2}^{+}, \mathrm{HD}^{+}$, and $\mathrm{D}_{2}^{+}$, , Phys. Rev. A 89 (5), 052521 (2014).

[9] Bakalov, D. and Schiller, S., "The electric quadrupole moment of molecular hydrogen ions and their potential for a molecular ion clock,” Appl. Phys. B 114 (1-2), 213-230 (2014).

[10] Leach, C.A. and Moss, R.E., "Spectroscopy and quantum mechanics of the hydrogen molecular cation: A test of molecular quantum mechanics,” Annu. Rev. Phys. Chem. 46, 55-82 (1995).

[11] Koelemeij, J.C.J., Roth, B., Wicht, A., Ernsting, I. and Schiller, S., "Vibrational spectroscopy of HD $^{+}$with 2-ppb accuracy," Phys. Rev. Lett. 98 (17), 173002 (2007).

[12] Bressel, U., Borodin, A., Shen, J., Hansen, M., Ernsting, I. and Schiller, S., "Manipulation of individual hyperfine states in cold trapped molecular ions and application to $\mathrm{HD}^{+}$frequency metrology," Phys. Rev. Lett. 108 (18), 183003 (2012). 
[13] Biesheuvel, J., Karr, J.-Ph., Hilico, L., Eikema, K.S.E., Ubachs W. and Koelemeij, J.C.J., "Probing QED and fundamental constants through laser spectroscopy of vibrational transitions in HD ${ }^{+}$, Nat. Commun. 7, 10385 (2016).

[14] Alighanbari, S., Giri, G.S., Constantin, F.L., Korobov, V. and Schiller, S., "Precise test of quantum electrodynamics and determination of fundamental constants with $\mathrm{HD}^{+}$ions," Nature 581, $152-158(2020)$.

[15] Patra, S., et al, "Proton-electron mass ratio from laser spectroscopy of $\mathrm{HD}^{+}$at the part-pertrillion level," Science 369 (6508), 1238-1241 (2020).

[16] Moss, R.E., "Calculations for vibration-rotation levels of $\mathrm{HD}^{+}$, in particular for high N," Mol. Phys. 78 (2), 371-405 (1993).

[17] Bakalov, D., Korobov, V.I. and Schiller, S., "High-precision calculation of the hyperfine structure of the HD' ion," Phys. Rev. Lett. 97 (24), 243001 (2006).

[18] Constantin, F.L., "Sensing electromagnetic fields with the ac-Stark effect in two-photon spectroscopy of cold trapped HD ${ }^{+}$Proc. SPIE 11347, 113470J (2020).

[19] Amitay, Z., Zajfman, D. and Forck, P., "Rotational and vibrational lifetime of isotopically asymmetrized homonuclear diatomic molecular ions," Phys. Rev. A 50 (3), 2304-2308 (1994).

[20] Bakalov, D. and Schiller, S., "Static Stark effect in the molecular ion HD"," Hyperfine Interact. $210(1-3), \quad 25-31(2012)$.

[21] Constantin, F.L., "Double-resonance two-photon spectroscopy of hydrogen molecular ions for improved determination of fundamental constants," IEEE Trans. Instrum. Meas. 68 (6), 2151-2159 (2019).

[22] Ludlow, A.D., Boyd, M.M., Ye, J., Peik, E. and Schmidt, P.O., “Optical atomic clocks,” Rev. Mod. Phys. 87 (2), 637 (2015).

[23] Schneider, T., Roth, B., Duncker, H., Ernsting, I. and Schiller, S., “All-optical preparation of molecular ions in the rovibrational ground state," Nat. Phys. 6, 275-278 (2010).

[24] Constantin, F.L., "Quantum state preparation, probing and detection of trapped HD' ions for precision measurements with two-photon transitions," Quantum Technology International Conference, 105 (2018).

[25] Itano, W.M., "External-field shifts of the ${ }^{199} \mathrm{Hg}^{+}$optical frequency standard,” J. Res. Natl. Inst. Stand. Technol. 105 (6), 829-837 (2000). 
[26] Karr, J. Ph, Kilic, S. and Hilico, L, "Energy levels and two-photon transition probabilities in the HD $^{+}$ion,” J. Phys. B 38 (7), 853-866 (2005).

[27] Berkeland, D.J., Miller, J.D., Bergquist, J.C., Itano, W.M. and Wineland, D.J., "Minimization of ion micromotion in Paul trap,” J. Appl. Phys. 83 (10), 5025-5033 (1998).

[28] Oskay, W.H., et al, "Single-atom optical clock with high accuracy," Phys. Rev. Lett. 97 (2), 020801 (2006).

[29] Schiller, S., Bakalov, D. and Korobov, V.I., "Simplest molecules as candidates for precise optical clocks," Phys. Rev. Lett. 113 (2), 023004 (2014).

[30] Koepsell, J., Thiele, T., Deiglmayr, J., Wallraff, A. and Merkt, F., "Measuring the polarization of electromagnetic fields using Rabi-rate measurements with spatial resolution : Experiment and theory," Phys. Rev. A 95 (5), 053860 (2017).

[31] Thiele, T., Lin, Y., Brown, M.O. and Regal, C.A., "Self-calibrating vector atomic magnetometry through microwave polarization reconstruction," Phys. Rev. Lett. 121 (15), 153202 (2018).

[32] Tiesinga, E., Mohr, P.J., Newell, D.B. and Taylor, B.N., "Values of fundamental physical constants. NIST," https://physics.nist.gov/cuu/Constants/index.html (2019). 


\section{Figure captions}

Figure 1. a. The experimental setup with the coordinate frames. $\mathrm{Be}^{+}$ions are cooled with the $313 \mathrm{~nm}$ laser (magenta line). Two-photon spectroscopy of $\mathrm{HD}^{+}$is performed with a stationary wave from the IR laser (red line). The detection is performed by dissociating $\mathrm{HD}^{+}$ions with the $175 \mathrm{~nm}$ laser (green line). The static magnetic field in the ion trap (olive line) can be oriented to any direction, defined with Euler angles $\alpha$ and $\beta$ in the Cartesian Laboratory Coordinate Frame (x,y,z) (black lines) ( $L C F)$. The THz-wave (blue line), off-resonantly coupled to the energy levels of the $\mathrm{HD}^{+}$ions, is linearly polarized and the electric field $\overrightarrow{\mathrm{E}}_{\mathrm{THz}}$ is oriented in a direction defined with the Euler angles $\theta$ and $\phi$ in the $L C F$. The standard components of the $\mathrm{THz}$ electric field are referenced in the Cartesian Molecular Ion Coordinate Frame $\left(\mathrm{x}_{\mathrm{c}}, \mathrm{y}_{\mathrm{c}}, \mathrm{z}_{\mathrm{c}}\right)$ (black lines) $(M I C F)$. The $M I C F$ is related to the $L C F$ by a $\mathrm{z}-\mathrm{y}-\mathrm{z}$ rotation with the Euler angles $(\alpha, \beta, \chi=0)$. b. Rotation-vibration energy levels of $\mathrm{HD}^{+}$ addressed in the $\mathrm{THz}$ sensing scheme. The two-photon rovibrational transitions are indicated in red, the dissociation in green, the THz-wave-driven electric dipole couplings in blue.
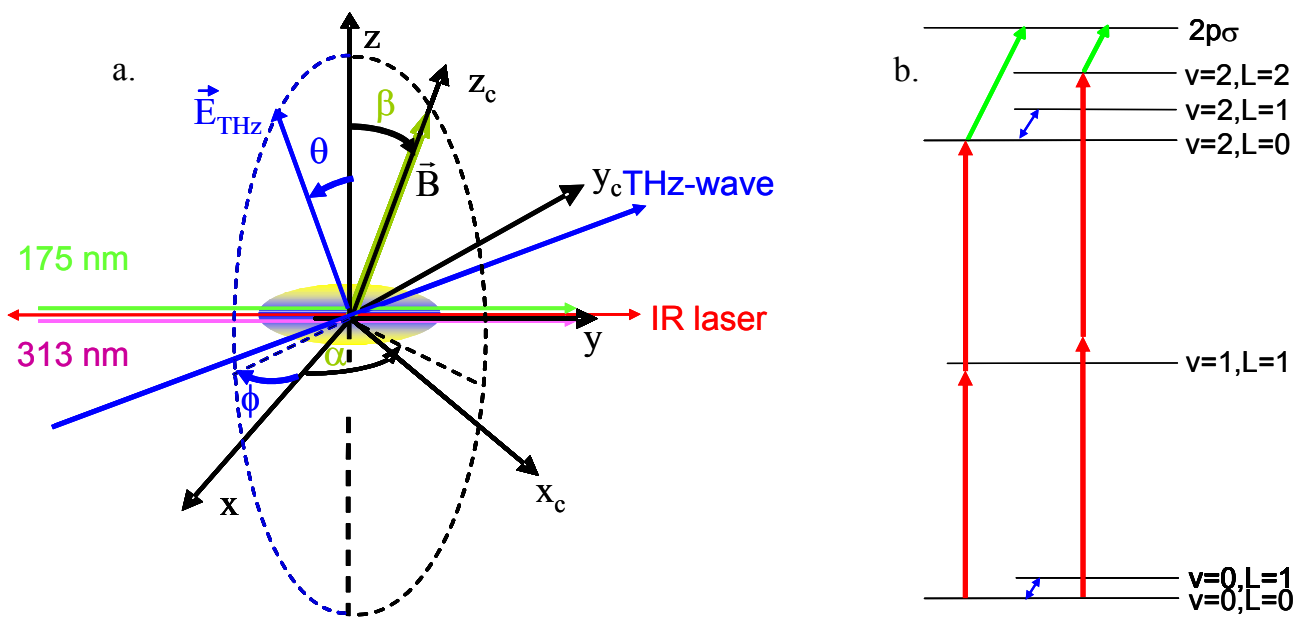

Figure 2. Dependence of the uncertainty of the magnetic field determined by Zeeman spectroscopy of the two-photon transition $\left(\mathrm{v}, \mathrm{L}, \mathrm{F}, \mathrm{S}, \mathrm{J}, \mathrm{J}_{\mathrm{Z}}\right)=(0,0,1,2,2,-2) \rightarrow(2,2,1,2,4,0)$ in function of the magnetic field strength.

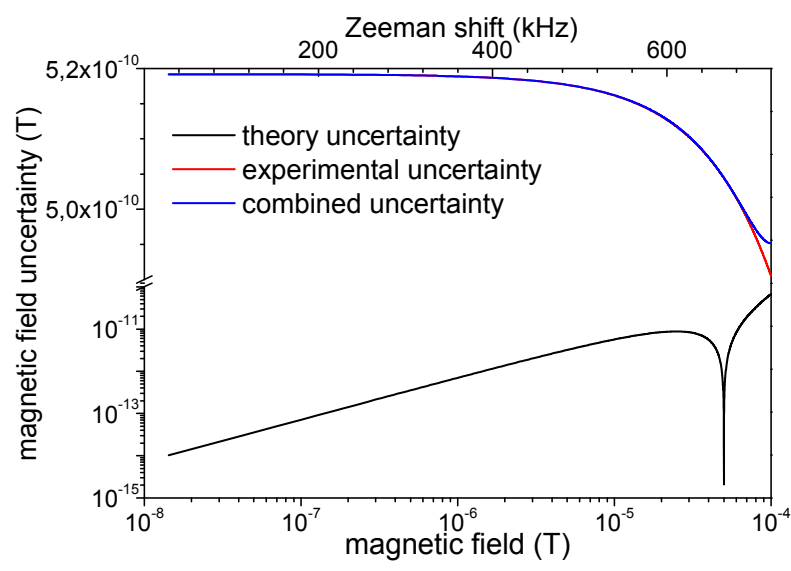


Figure 3. Uncertainties of the THz-wave electric field in function of the THz-wave electric field : combined experimental and theory errors (continuous red line) and the contribution from the experimental errors (dotted blue line).

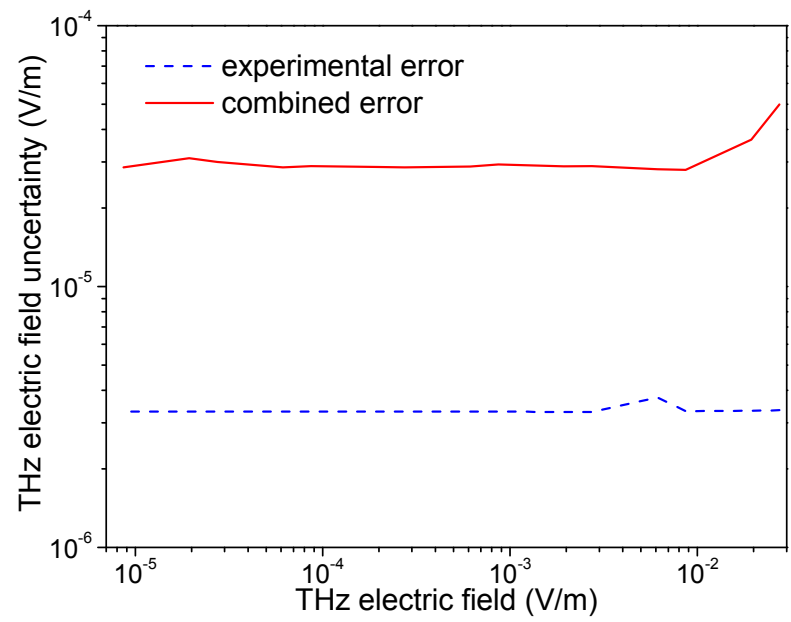

Figure 4. Uncertainties of the amplitudes and phases of the Cartesian components of the electric field of a THz-wave derived from lightshift measurements in function of the orientation of the polarization vector. (a) Uncertainty of $\mathrm{E}_{\mathrm{x}}$. (b) Uncertainty of $\mathrm{E}_{\mathrm{y}}$. (c) Uncertainty of $\mathrm{E}_{\mathrm{z}}$. (d) Uncertainty of $\varphi_{\mathrm{x}}$. (e) Uncertainty of $\varphi_{\mathrm{y}}$.

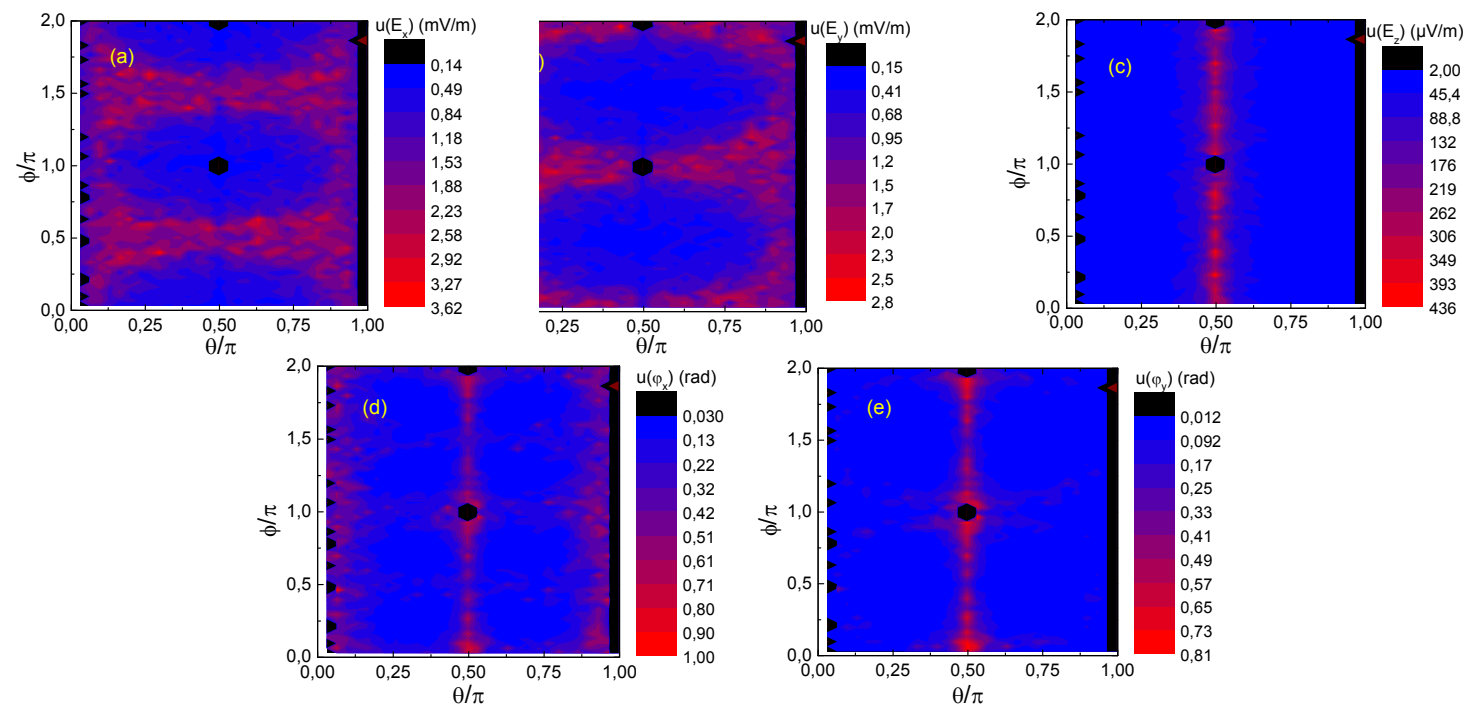


Figure 5. Uncertainties of the THz-wave parameters determined from lightshift measurements in function of the amplitude of the $\mathrm{THz}$ electric field.

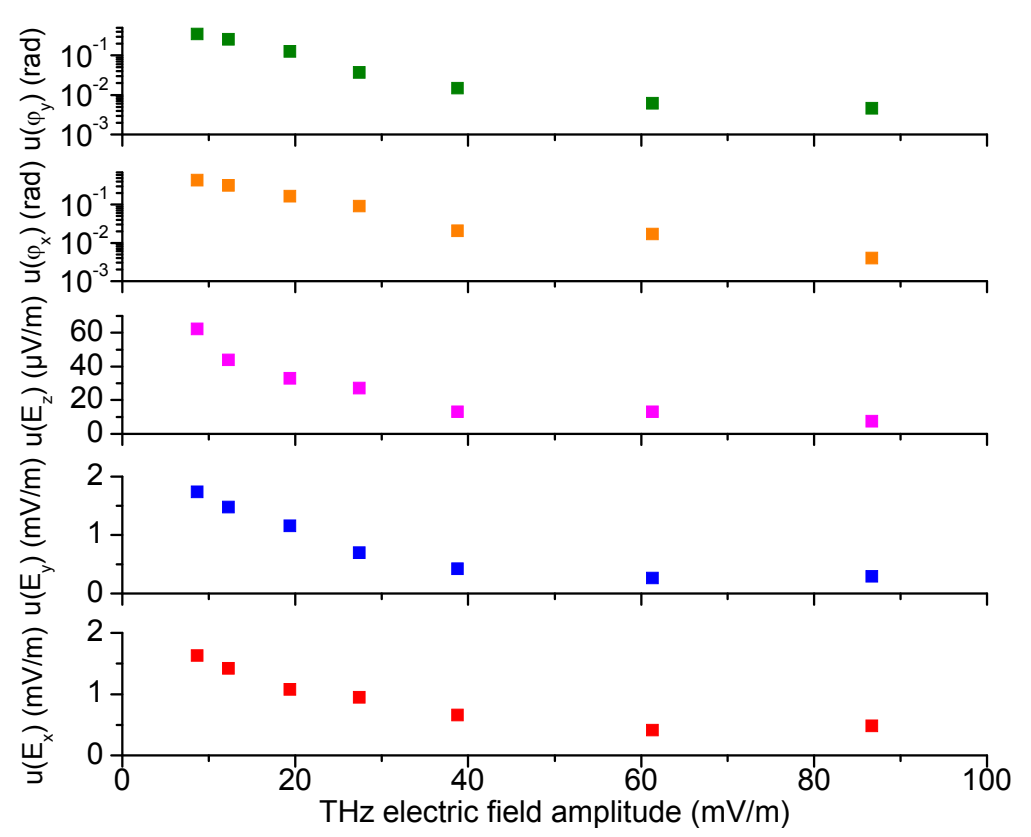

Table captions

Table 1. Systematic frequency shifts and uncertainties for $\mathrm{HD}^{+}$two-photon transitions.

Transition

$\left(v_{1}, L_{1}, F_{1}, S_{1}, J_{1}, J_{z 1}\right) \rightarrow\left(v_{2}, L_{2}, F_{2}, S_{2}, J_{2}, J_{z 2}\right)$

Frequency

Systematic shift

Zeeman shift

Trap shift

Second-order Doppler shift

Blackbody radiation shift

Quadrupole shift

Lightshift from the spectroscopy laser

Lightshift from the cooling laser

Lightshift from the dissociation laser
(1)

$(0,0,1,2,2,2) \rightarrow(2,0,1,2,2,2)$

$55.909 \mathrm{THz}$

Shift $(\mathrm{Hz}) \quad$ Uncertainty $(\mathrm{Hz})$

$<2 \times 10^{-2}$

$-45$

$-2$

$-3.3 \times 10^{-3}$

0

$-1.1$

$-1.3 \times 10^{-3}$

$-0.1$

$-48.2$

$2 \times 10^{-2}$

4.1

$18 \times 10^{-2}$

$3 \times 10^{-4}$

$$
0
$$

$1.1 \times 10^{-2}$

$1.3 \times 10^{-5}$

$1 \times 10^{-3}$

4.1

$(0,0,1,2,2,-2) \rightarrow(2,2,1,2,4,0)$

$57.701 \mathrm{THz}$

Shift $(\mathrm{Hz}) \quad$ Uncertainty $(\mathrm{Hz})$

13992.8

0.58 14

$5.2 \times 10^{-2}$

$-7.3 \times 10^{-2}$

$-7.8 \times 10^{-3}$

$6.6 \times 10^{-3}$

$4 \times 10^{-4}$

$-1.7$

0.47

0.56

$5.6 \times 10^{-3}$

$-1.3 \times 10^{-3} \quad 1.3 \times 10^{-5}$

$-0.1$

13992.1

$1 \times 10^{-3}$ 